\title{
Resource Availability Based Assortment Of Generating Micro Grid Power Using Hybrid Algorithm
}

\author{
D.Santhosh Kumar, M.Kalyanasudaram, V.Lavanya
}

\begin{abstract}
The levels of fundamental power generation from non-renewable resources such as coal, nuclear reactors are in less measure around world. Most of the power grids are based on non-renewable resources. The frame work and cost effective of such grids are more expensive. The economical level is increasing while increasing power generating ratios.So that we alter the method that is to generate the power from renewable resource such as forming solar (photo Voltaic) station. The system focused towards connecting the all energy resources like renewable as well as non- renewable sources and stores the energy. The energy distributed from the storage units to all distributed networks. The power generations of the grids are based on availability of resource. With respect to time, the total availability of resources is analyzed from each of the micro grids. The operation of this system is based on BFO ((Bacterial Foraging Optimization))algorithm which verifies all the sources required for power generation as well as requirement of energy demand and priority will be given to the resource highly available with cost effective in to considerations.
\end{abstract}

Index Terms - Renewable Energy (Solar), Non - Renewable Energy (Diesel generator), BFO (Bacterial Foraging Optimization) algorithm

\section{INTRODUCTION}

A Microgrid refers to distributed energy resources and loads that can be operated in a controlled, coordinated way; they can be connected to the main power grid, operate in "islanded" mode or be completely off-grid. Microgrids are low- or medium-voltage grids located at or near the consumption sites. They can generate power from both renewable and conventional sources and although they are mainly electrical systems, they can also incorporate a thermal energy component, such as combined heat and power. Microgrids are increasingly being equipped with energy storage systems, as batteries become more cost competitive. The system is controlled through a Microgrid controller incorporating demand-response so that demand can be matched to available supply in the safest and most optimized manner. A flywheel or battery-based grid stabilizing system can be included to offer real and reactive power support.

D.Santhosh Kumar, Assistant Professor Of Electrical And Electronic Engineering, Vivekanandha College Of Engineering For Women [Autonomous], Tiruchengode

D.Santhosh Kumar, Assistant Professor Of Electrical And Electronic Engineering, Vivekanandha College Of Engineering For Women [Autonomous], Tiruchengode

M.Kalyana Sundaram , Assistant Professor Of Electrical And Electronic Engineering, Vivekanandha College Of Engineering For Women [Autonomous], Tiruchengode

V.Lavanya Pg Scholar., M.E In Vivekanandha College Of Engineering Women [Autonomous]
The concept of a Microgrid is not new: the earliest electricity networks were essentially Microgrids before they were joined into regional and national grids. What is new is their changing and expanding role, in the face of rising power demands, the falling cost of renewable sources, and the increasing need for supply resilience and autonomy - both on- and off-grid. A Microgrid is a localized group of electricity sources and loads that normally operates connected to and synchronous with the traditional wide area synchronous grid (macrogrid), but can also disconnect to "island mode" — and function autonomously as physical or economic conditions dictate. In this way, a Microgrid can effectively integrate various sources of distributed generation (DG), especially Renewable Energy Sources (RES) - renewable electricity, and can supply emergency power, changing between island and connected modes. Control and protection are challenges to Microgrids. A very important feature is also to provide multiple end-use needs as heating, cooling, and electricity at the same time since this allows energy carrier substitution and increased energy efficiency due to waste heat utilization for heating, domestic hot water, and cooling purposes (cross sectoral energy usage).

The hybrid energy system consisting of two or more type of energy sources has ability to reduces BES requirements and increase reliability by using MPPT technique. The solar power is produced for the cost effective way. Solar MPPT control strategy to give maximum power by solar energy [1]. The microgrid is fluctuating output from renewable energy sources can cause oscillations in the DC bus voltage. The power quality on the AC side is maintained [2]. The different operating regions with in the domain of stability are defined based on the sensitivity analyses and improve the stability for the microgrids is determined [3]. Elimination problem is reduced from both hybrid generations with the storage. An objective function of the concept is to minimize the cost and improve the reliability with curtail Green House Gases (GHG) emission [4]. Elimination of the battery charge is also a circuit parameter for management in the power and energy flow in the system will supplied with multiple power supply and electro chemical batteries. [5] PID controllers has been designed and tuned in the order to avoid the intervention of the protection relays of the battery inverters. Stand alone system in the micro grid integrated with an energy storage is followed by the Energy Management System on the battery bank side. It will improve the system efficiency and minimize the operation cost of the system [6]. Management issues and the power flow control among multiple source dispersed entire AC and DC system. The hybrid system in microgrid allows the different AC or DC loads in the sources to be more 
flexible. It is flexible in order reduces the system cost and the efficiency of the power in hybrid system [7].

Monitoring and control the system for alternative distribution grid system based on the energy storage systems. Digital processing chain relies on the Goertzel algorithm in order to obtain the grid voltages and current in sequence [8]. System has a relative low sudden for communication network. It can be scaled to a higher number of nodes. In AC and DC microgrids hybrid for the improved integration of photo voltaic with battery storage. Power conversion losses can be reduced to $20-30 \%$ in the residential side with net zero energy balance. Photo voltaic and battery size important and the parameters for the improved system performance [9]. In hybrid system single phase and three phase micro grid the power coordinated control method is used to support the frequency capability. [10] In this photo electric vehicle can provide frequency in hybrid is reduce the $\mathrm{AC}$ bus voltage fluctuation by providing the real and reactive power support.

\section{HYBRID ENERGY SYSTEM:}

Hybrid energy system is combined both renewable (solar) and non renewable (diesel generator) energy sources given to the load. It can design to extract power by using both energy sources . when using this system the cost is low, reliability is good, higher efficiency and less emission. when comparing other renewable energy resources the wind and solar have good availability and the cost is less. and no need to find out the special location to installed this system.

\section{A. .SOLAR (PHOTOVOLTAIC CELL):}

Solar energy is an renewable energy source that energy will gets by the radiation of the sun. It is an present on the earth continuously the energy will be stored and in an abundant manner. Solar energy is freely available. It doesn't produce any polluted gases and it is low maintenance cost. Only problem in solar system it dose not stored the power in bad weather condition. But the efficiency will be higher when compare to other energy sources. It only need initial investment. Life time will be high and it generate lower emission.

\section{B. DIESEL GENERATOR}

In this system they combined the diesel engine with an electric generator (often an alternator) to generate the electric energy. In this system they are usually designed to run on diesel fuel but some times it change for other natural gas.Diesel generator it does not connect to the grid it is an emergency power-supply when the grid power is failed as well as it is used in rare condition such as peak lopping, grid support and it exported to the main grid. Proper sizing of diesel generators it is critical to avoid low-load.it is an Non linear load and its size ranges is around 50MW and above, diesel generator is an open cycle gas turbine and the efficiency is more at full load condition. But it is not renewable energy resource.

\section{FUNCTIONAL BLOCK DIAGRAM}

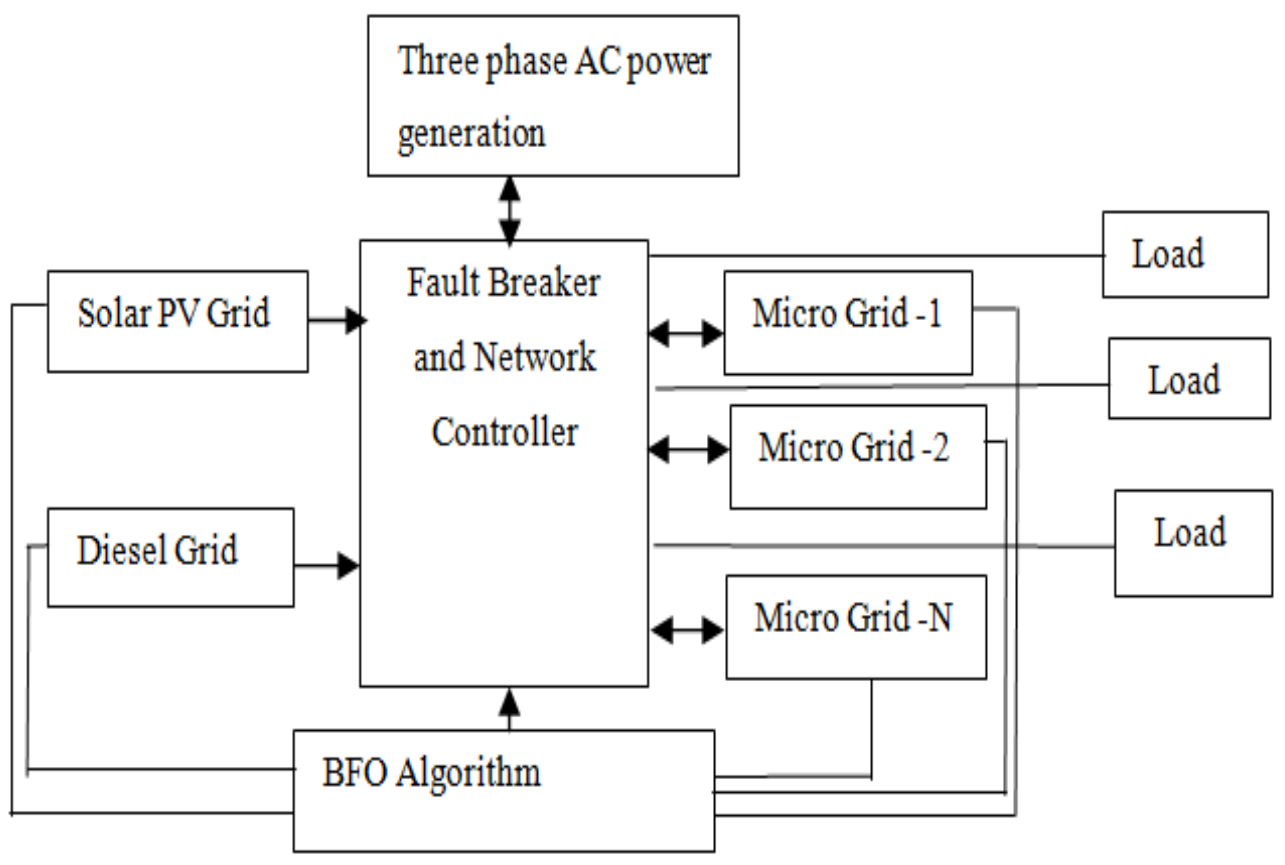

Figure.1.Functional Block Diagram of the System 
This system consists of ' $\mathrm{N}$ ' number of Microgrids, power grids, destination i.e., loads, switching exchange centre and resource calibrator. The operation of the calibrator is based on BFO (Bacterial foraging optimization) algorithm because; this is the cost effective with cleverer optimization algorithm. The resource calibrator is the control unit of the system which can sense the power parameters of each Microgrid as well as power grid and enables the distribution links in switching exchange centre between the Microgrids with power grid when the source of the power grid is failure or decreasing the resource power level of the grid.

Based on this collaborative and optimized power link between the grids, there is continuous power supplied to the destination can be increase the power quality of the distribution network. The BFO algorithm is briefly explained below the topic.

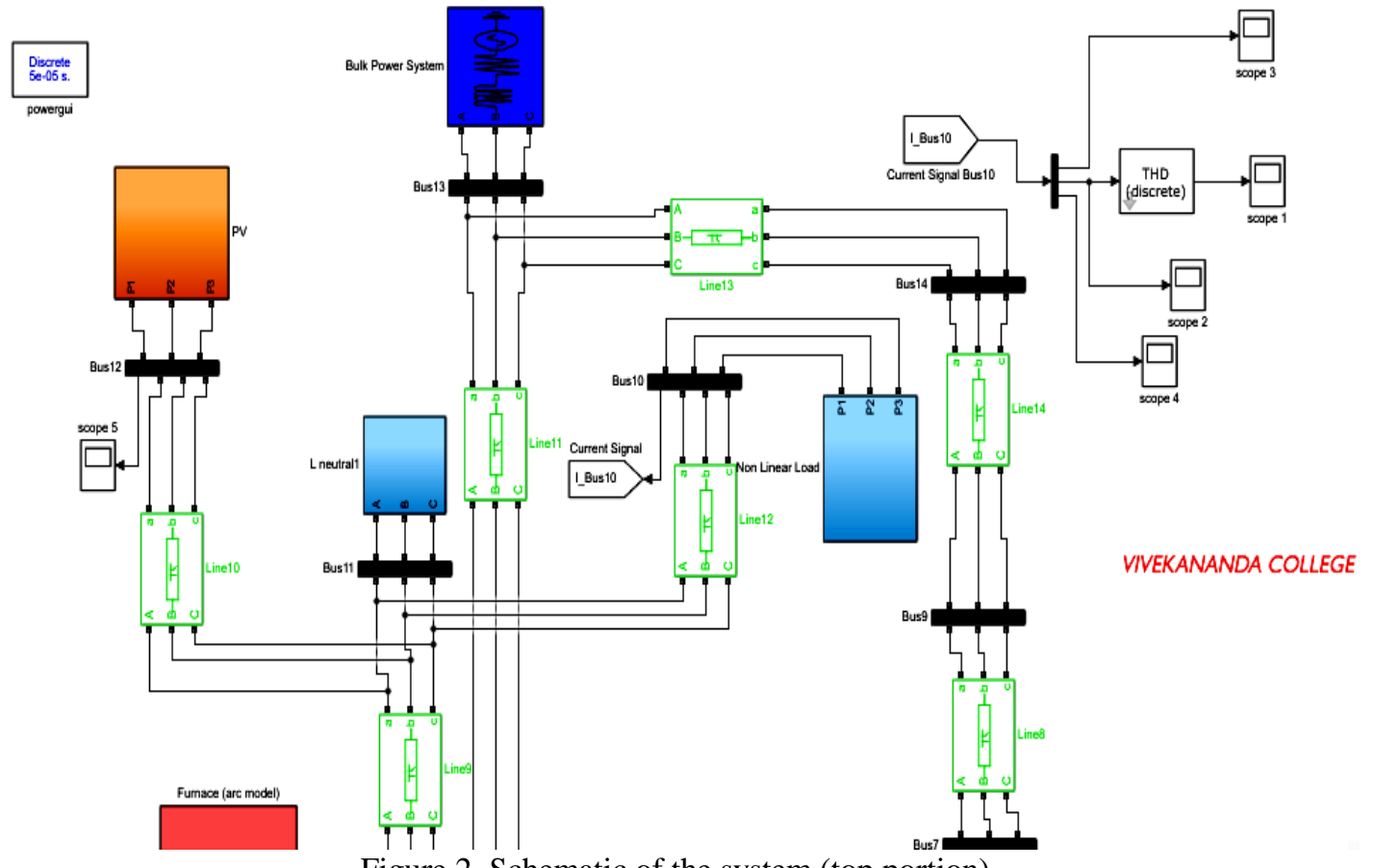

Figure.2. Schematic of the system (top portion)

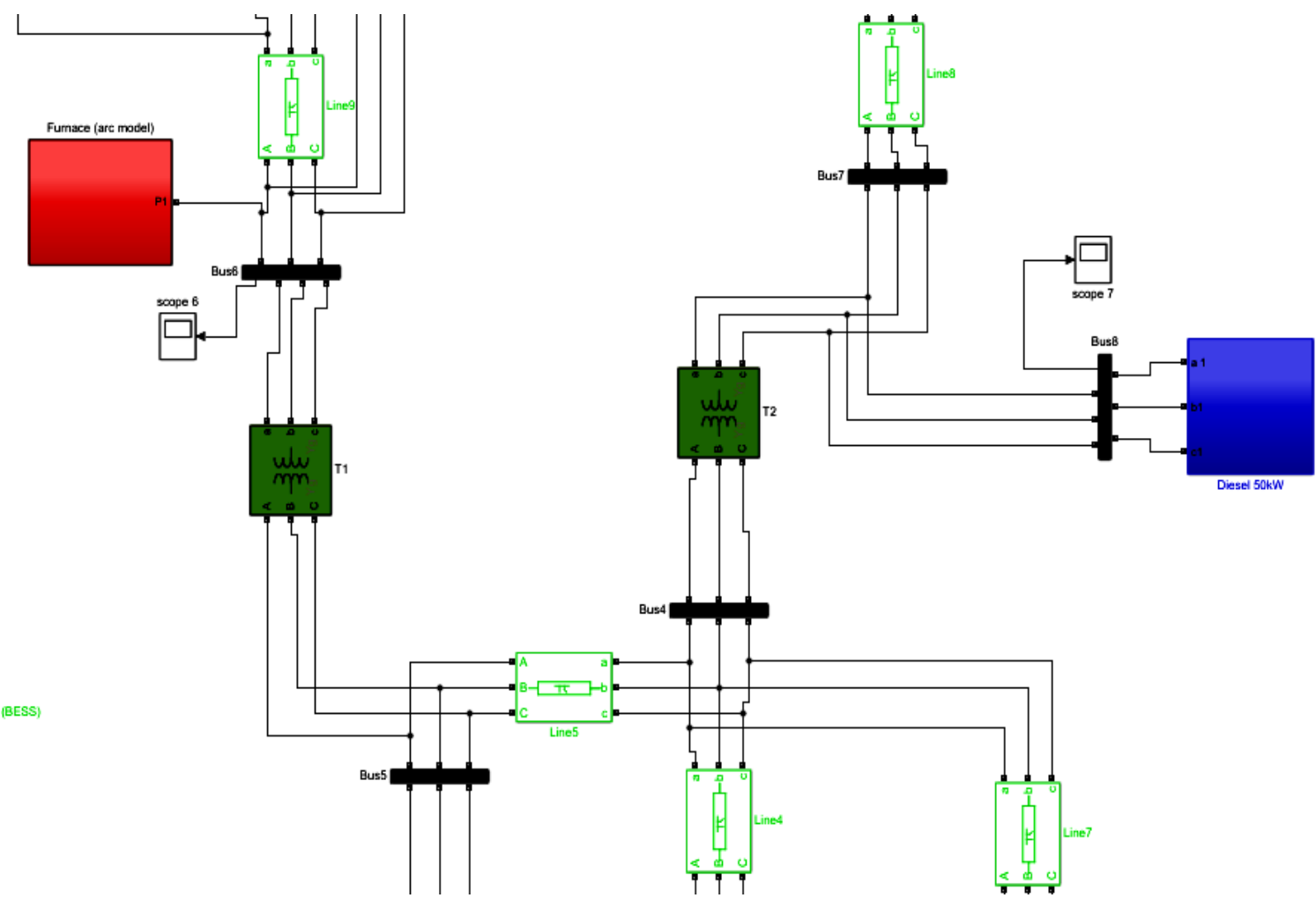

Figure.2.Schematic of the system (middle portion) 


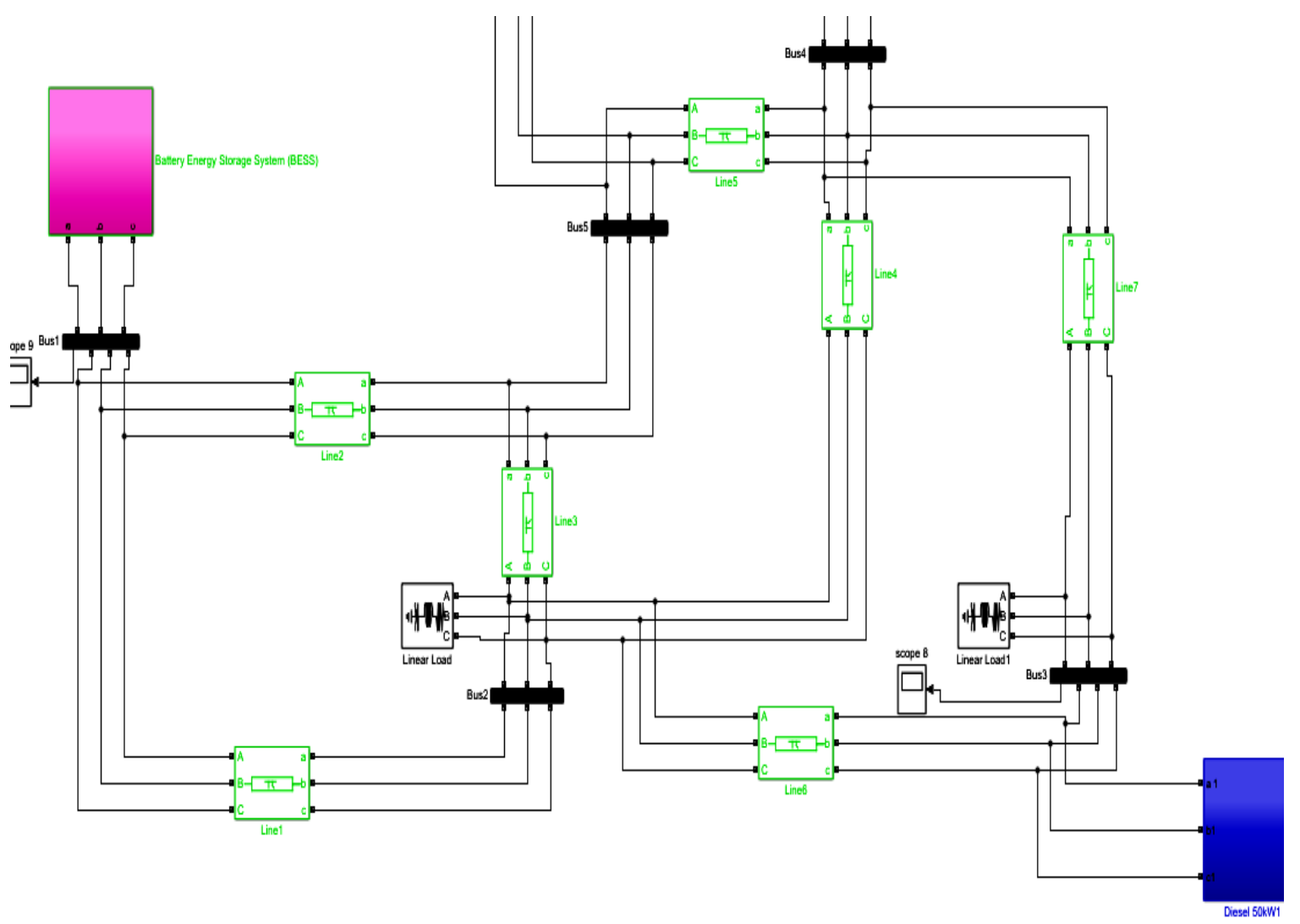

Figure.2.Schematic of the system (bottom portion)

\section{BFO ALGORITHM:}

Bacterial Foraging Optimization Algorithm (BFO) has been widely accepted as a global optimization algorithm of current interest for distributed optimization and control. BFOA is inspired by the social foraging behavior of Escherichia coli. BFO has already drawn the attention of researchers because of its efficiency in solving real-world optimization problems arising in several application domains. The underlying biology behind the foraging strategy of E.coli is emulated in an extraordinary manner and used as a simple optimization algorithm.

Bacteria Foraging Optimization Algorithm (BFO), proposed by Passino, is a new comer to the family of nature-inspired optimization algorithms. For over the last five decades, optimization algorithms like Genetic Algorithms (GAs), Evolutionary Programming (EP), Evolutionary Strategies (ES), which draw their inspiration from evolution and natural genetics, have been dominating the realm of optimization algorithms. Recently natural swarm inspired algorithms like Particle Swarm Optimization (PSO), Ant Colony Optimization (ACO) have found their way into this domain and proved their effectiveness. Following the same trend of swarm-based algorithms, Passino proposed the BFO. Application of group foraging strategy of a swarm of E.coli bacteria in multi-optimal function optimization is the key idea of the new algorithm. Bacteria search for nutrients in a manner to maximize energy obtained per unit time. Individual bacterium also communicates with others by sending signals. A bacterium takes foraging decisions after considering two previous factors. The process, in which a bacterium moves by taking small steps while searching for nutrients, is called chemotaxis and key idea of BFOA is mimicking chemotactic movement of virtual bacteria in the problem search space.

\section{BFO Algorithm:}

\section{Parameters:}

[Step 1] Initialize parameters p, S, Nc, Ns, Nre, Ned, Ped, $\mathrm{C}(\mathrm{i})(\mathrm{i}=1,2 \ldots \mathrm{S}), \theta^{\mathrm{i}}$.

\section{Algorithm:}

[Step 2] Elimination-dispersal loop: $1=1+1$

[Step 3] Reproduction loop: $\mathrm{k}=\mathrm{k}+1$

[Step 4] Chemotaxis loop: $\mathrm{j}=\mathrm{j}+1$

[Step 5] If $\mathrm{j}<\mathrm{N}_{\mathrm{c}}$, go to step 4 . In this case continue chemotaxis since the life of the bacteria is not over.

[Step 6] Reproduction:

[a] For the given $\mathrm{k}$ and $\mathrm{l}$, and for each $\mathrm{i}=1,2, \ldots, \mathrm{S}$, let

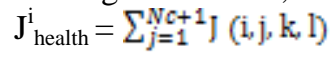

be the health of the bacterium i (a measure of how many nutrients it got over its lifetime and how successful it was at avoiding noxious substances). Sort bacteria and chemotactic parameters $\mathrm{C}(\mathrm{i})$ in order of ascending cost $\mathbf{J}_{\text {health }}$ (higher cost means lower health).

[b] The $S_{\mathrm{r}}$ bacteria with the highest $\mathrm{J}_{\text {health }}$ values die and the remaining $S_{\mathrm{r}}$ bacteria with the best values split (this process is performed by the copies that are made are placed at the same location as their parent).

[Step 7] If $\mathrm{k}<\mathrm{N}_{\mathrm{re}}$, go to step 3. In this case, we have not reached the number of specified reproduction steps, so we start the next generation of the chemotactic loop.

[Step 8] Elimination-dispersal: For $\mathrm{i}=1,2 \ldots, \mathrm{S}$ with probability $\mathrm{P}_{\mathrm{ed}}$, eliminate and disperse each bacterium (this keeps the number of bacteria in the population constant). To do this, if a bacterium is eliminated, simply disperse another one to a random location on the optimization domain. If $1<$ $\mathrm{N}_{\mathrm{ed}}$, then go to step 2; otherwise end. 
FLOWCHART OF BFO:

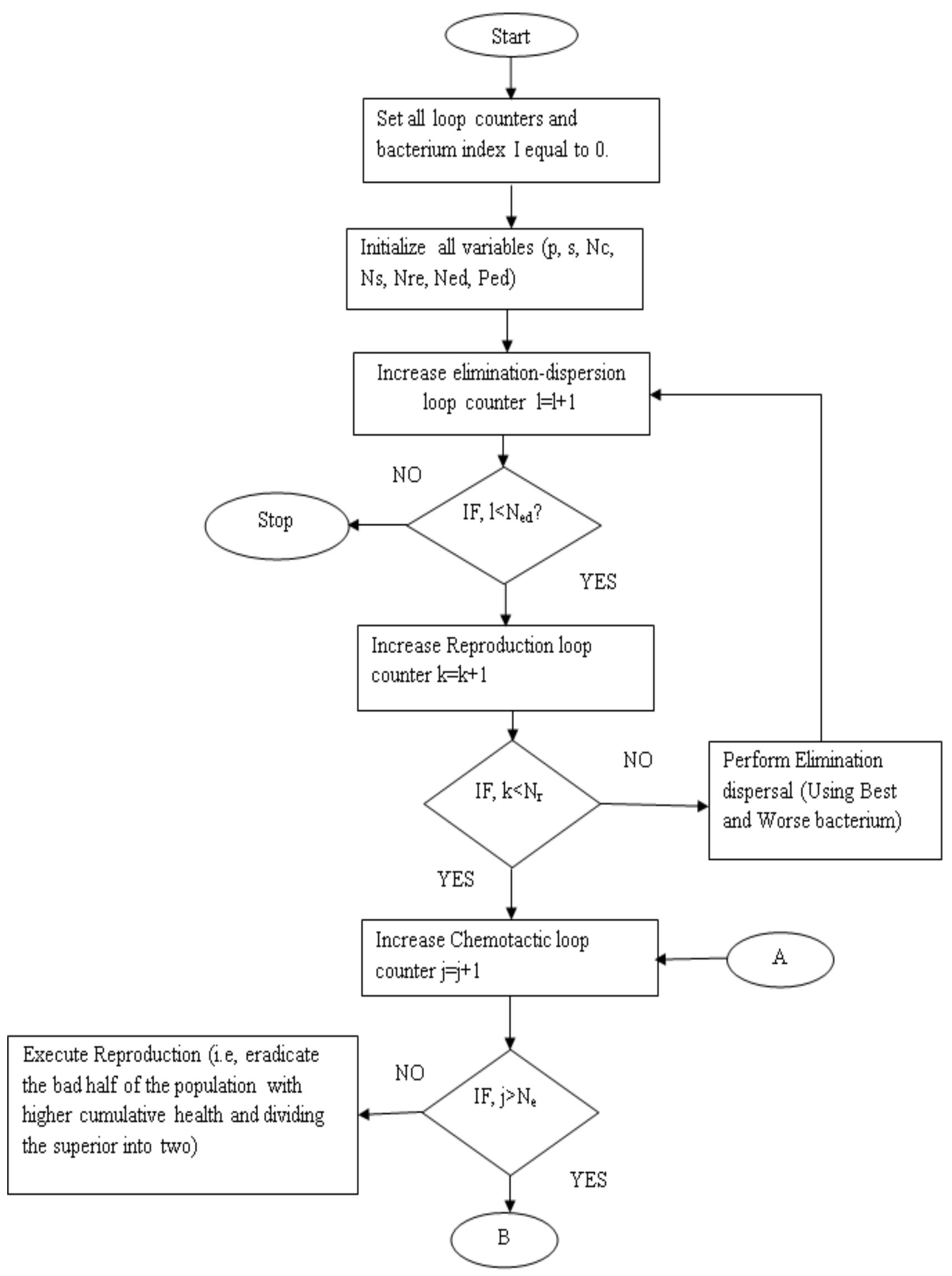




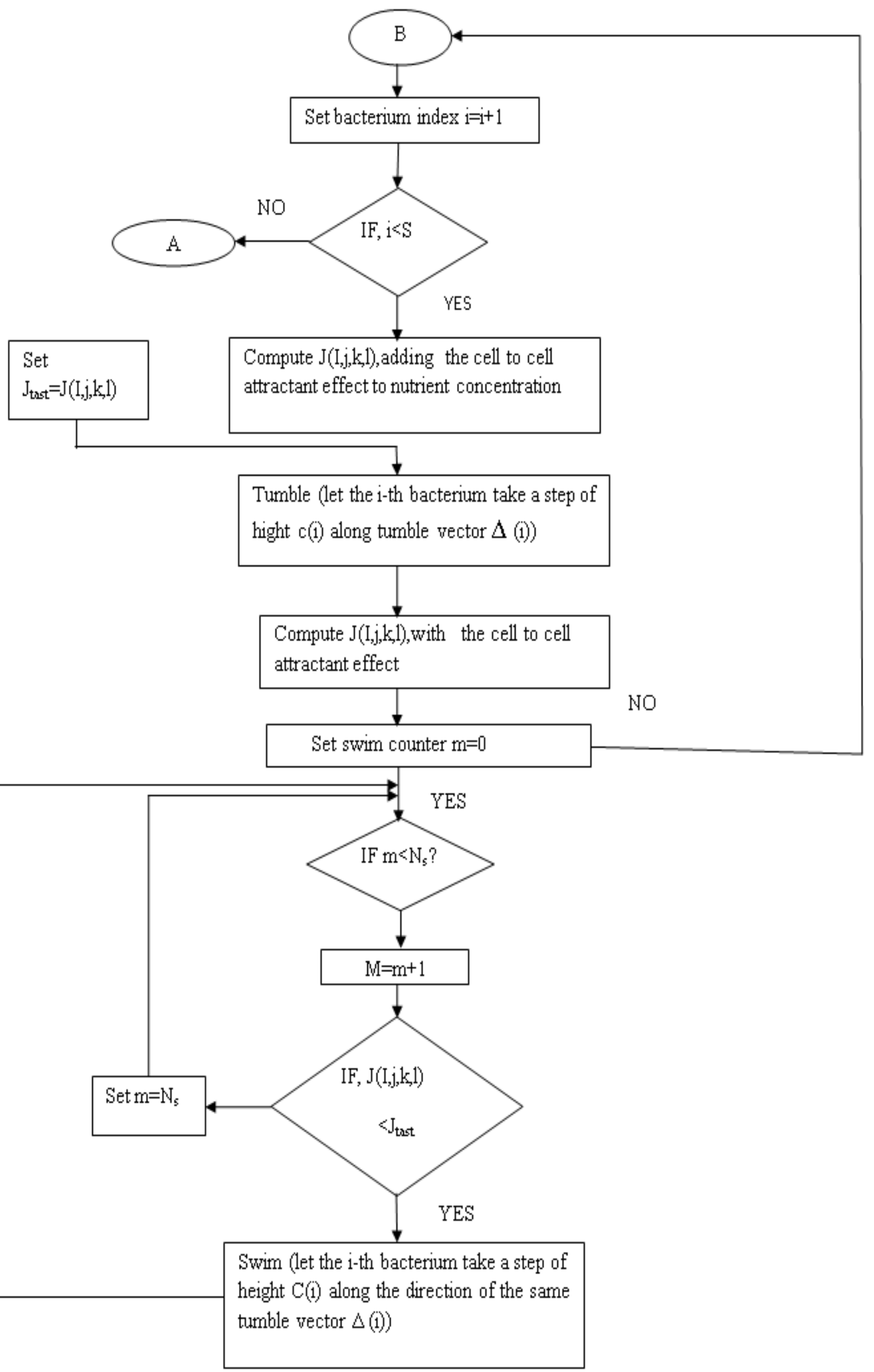

Figure.3.Flow Chart of Bacterial Foraging Optimization Algorithm 


\section{SIMULATION RESULTS:}

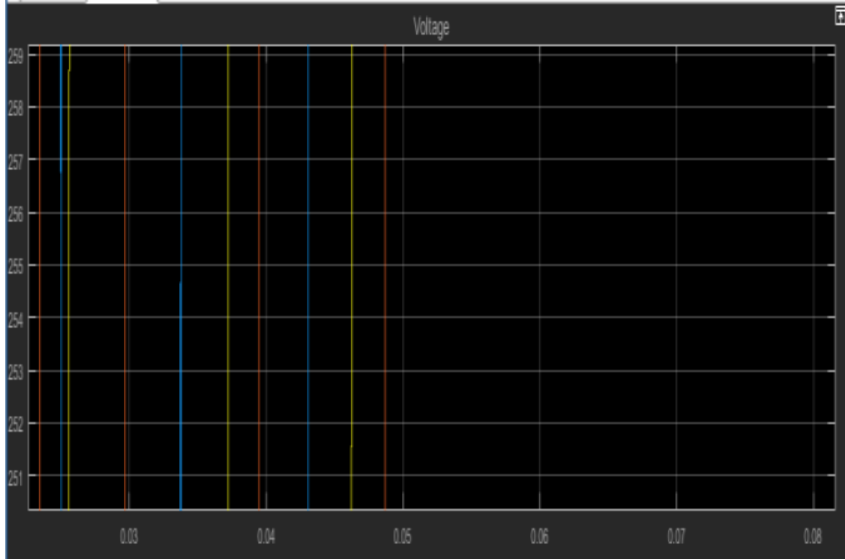

Figure.4. Output of the PV System

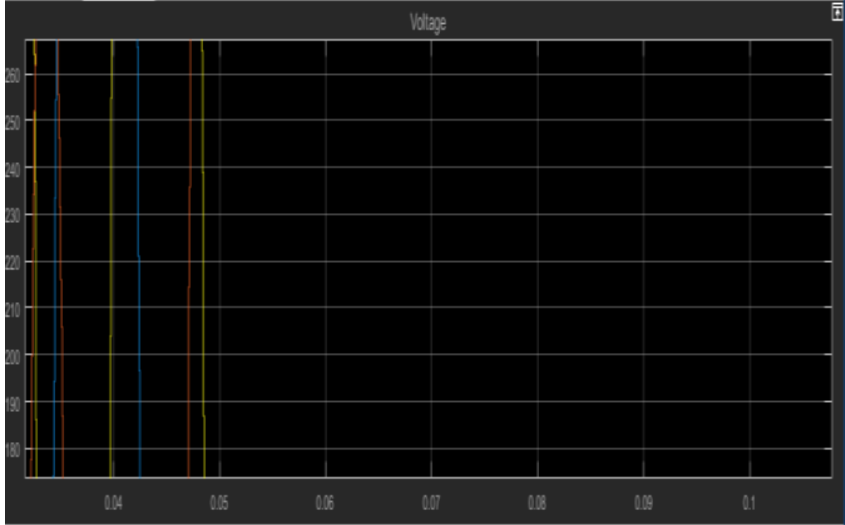

Figure.5. Output of the Diesel Generator

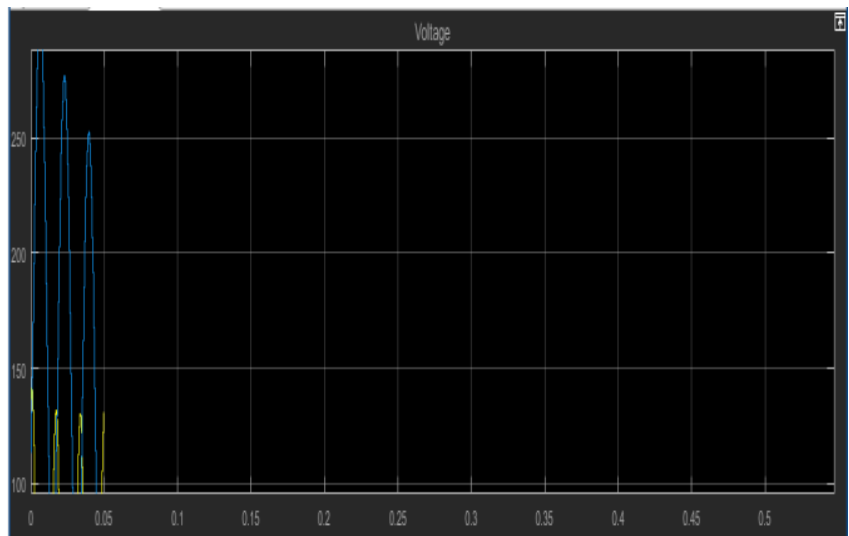

Figure.6. Output of the Bulk Power System

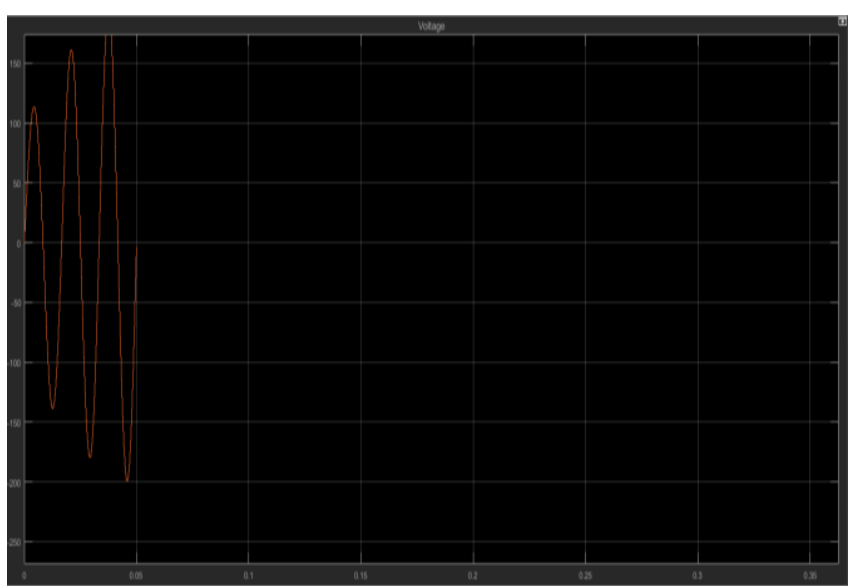

Figure.7. Output of the Battery Energy Storage System

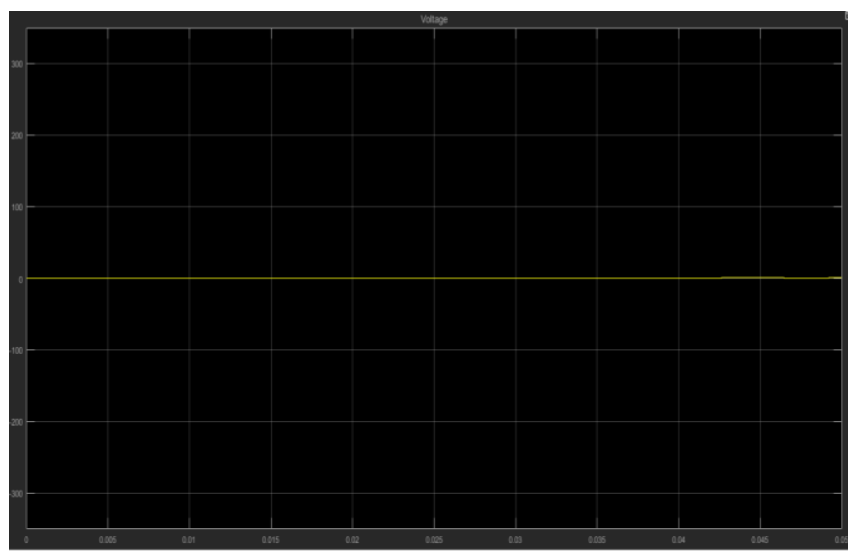

Figure.8. Output of the Total Harmonic Distortion System

\section{CONCLUSION:}

Optimal scheduling of power generation among different DERs in a microgrid needs a robust and well-organized optimizer which is able to escape local optima. This system proposes a new approach based on BFOA, called Bacterial foraging optimization algorithm, for optimally sharing the demanded power among DERs in a microgrid. BFO attempts to improve search capability of resource by increasing intensification and amplifying local and global searches. Demanded load is optimally shared by BFO among different microgrids by the aim of cost minimization. Simulated results reveal that results obtained by BFO are more accurate than results found by GA, PSOw and PSOcf. BFO not only finds the minimal generation cost but also solves the scheduling problem around $2 \mathrm{sec}$. Due to the stochastic nature of the power production by renewable resources, it is assumed that one PV plant of considered Microgrid is not able to generate power. In this case, performance of BFO is superior since it supplies the requested power by the remaining DERs at minimal cost compared to the other algorithms and satisfies the balance equation. As the operation of smart grids is heading towards real-time fully automated practices, the proposed technique can be much more desirable than conventional solutions. It can be dawn that $\mathrm{BFO}$ is an efficient optimization technique which may find better result than other optimizers for solving optimization problems related to Microgrid power scheduling and other power system optimization problems.

\section{REFERENCE:}

[1] Shailendra Kr. Tiwari, Bhim Singh \& Puneet K. Goel, " Design and Control of Micro-Grid fed by Renewable Energy Generating Sources", IEEE Transactions on Indistrial Application, 2017.

[2] Yinghao Shan, Jiefeng Hu, Zilin Li, \& Josep M., "AModel Predictive Control forRenewable Energy BasedAC Microgridswithout Any PID Regulators", IEEE Transactions on Power Electronics,2018.

[3] Adedayo Aderibole, Zeineldin H. H., Mohamed Shawky El-Moursi, Jimmy C. -H. Peng, , \& Mohamed Al Hosani, "Domain of Stability Characterization for Hybrid Microgrids Considering Different Power Sharing Conditions", IEEE Transactions on Energy Conversion,2017.

[4] Umer Akram, Muhammad Khalid \& Saifullah Shafiq, "An Innovative Hybrid Wind-Solar and Battery-Supercapacitor Microgrid System-Development and Optimization", IEEE Access,2017.

[5] Bruno Belvedere, Michele Bianchi, Alberto Borghetti, Carlo Alberto Nucci, Mario Paolone \& Antonio Peretto, "A Microcontroller-Based Power Management System for Standalone MicrogridsWith Hybrid Power Supply”, IEEE Transactions on Sustainable Energy,2012.

[6] Giorgio Cau, Daniele Cocco, Mario Petrollese, Søren Knudsen Kær \& Christian Milan, "Energy management strategy based on short-term 
generation scheduling for a renewable microgrid using a hydrogen storage system",EISEVIER,2014.

[7] Navid Eghtedarpour \& Ebrahim Farjah, "Power Control and Management in a Hybrid AC/DC Microgrid", IEEE Transactions on Smart Grid,2014.

[8] Pablo García, Pablo Arboleya, Bassam Mohamed, Abel Alberto Cuadrado Vega, \& Marcos Cuadrado Vega, "Implementation of a hybrid distributed/centralized real-time monitoring system for a DC/AC Microgrid with energy storage capabilities", IEEE Transactions on Industrial Informatics, 2016.

[9] Johannes Hofer, Bratislav Svetozarevic, Arno Schlueter, "Hybrid AC/DC Building Microgrid for Solar PV and Battery Storage Integration", IEEE Transactions,2017.

[10] Xiaoping Zhou, Yandong Chen , Leming Zhou, An Luo, Josep M Guerrero, Wenhua Wu, Ling Yang, Wenjuan Tan, "Power coordinated control method with frequency support capability for hybrid single/three-phase Microgrid",IET Journal,2017.

D.SANTHOSH KUMAR he received the Undergraduate Degree B.E. (Electrical and Electronics Engineering) in 2007 in Sona College of Technology from the Anna University.He completed his Master Degree M.E. (Instrumentation Engineering) in 2009 at Madras Institute of Technology from the Anna University. He is having 5 years in teaching field.He is a Assistant professor of Electrical and Electronic Engineering, Vivekanandha College of Engineering For Women [Autonomous],Tiruchengode.He has Published 24 papers in International, National Journals and Conference proceeding.

M.KALYANA SUNDARAM he received the Undergraduate Degree B.E. (Electrical and Electronics Engineering) in 2001 in V.M.K.V.Engineering College,Salem from the University of Madras. He completed his Master Degree M.E. (Power System Engineering) in 2004 at Annamalai University-Chidambaram. He Pursing his Ph.D degree(Electrical and Electronics Engineering) at Anna University-Chennai. He is having 13 years in teaching field.He is a Assistant professor of Electrical and Electronic Engineering, Vivekanandha College of Engineering For Women [Autonomous],Tiruchengode.He has Published 16 papers in International, National Journals and Conference proceeding.

V.LAVANYA PG scholar., M.E in Vivekanandha College of Engineering Women [Autonomous] (2017-2019),completed B.E [Electrical and Electronic Engineering] in Jayam College of Engineering and Technology with 70\%,HSC in John Britto Higher Secondary School with $65 \%$.Published 2papers in Journals 